\title{
Stimulatory Effects of Sublethal Doses of Dimethachlon on Sclerotinia sclerotiorum
}

Feng Zhou, Hong-Jie Liang, Ya-Li Di, Hong You, and Fu-Xing Zhu, College of Plant Science and Technology, Huazhong Agricultural University, Wuhan, 430070, China

\begin{abstract}
Zhou, F., Liang, H.-J., Di, Y.-L., You, H., and Zhu, F.-X. 2014. Stimulatory effects of sublethal doses of dimethachlon on Sclerotinia sclerotiorum. Plant Dis. 98:1364-1370.

Growth and virulence stimulations of sublethal doses of fungicides on plant-pathogenic fungi and oomycetes have been reported and the stimulatory effects are potentially relevant to plant disease management. Sclerotinia sclerotiorum is one of the most devastating and economically important necrotrophic fungal phytopathogens, capable of infecting more than 400 species of plants worldwide. In order to study stimulatory effects of sublethal doses of fungicides on S. sclerotiorum, 55 dimethachlon-sensitive isolates and 3 dimethachlon-resistant isolates of $S$. sclerotiorum were assayed to determine effects of sublethal doses of dimethachlon on mycelial growth rate on potato dextrose agar (PDA) media and virulence on oilseed rape plants. Results showed that all 3 dimethachlon-resistant isolates and 13 of the 55 sensitive isolates exhibited stimulatory responses to sublethal doses of dimethachlon.

Dimethachlon-resistant isolates grew significantly $(P<0.05)$ faster on PDA media amended with dimethachlon at 0.5 to $4 \mu \mathrm{g} / \mathrm{ml}$ than on fungicide-free PDA media. As for virulence on detached leaves of oilseed rape plants, lesion diameters of dimethachlon-resistant isolates after growth on PDA media amended with dimethachlon at 0.5 to 2 $\mu \mathrm{g} / \mathrm{ml}$ were significantly larger $(P<0.05)$ than the control. The maximum stimulatory effects were 42.40 to $59.80 \%$. In pot experiments, for both dimethachlon-sensitive and -resistant isolates, significant $(P<$ 0.05 ) virulence stimulations were observed after spraying with dimethachlon at a concentration of $2 \mu \mathrm{g} / \mathrm{ml}$. After growing on dimethachlon-amended PDA media, $\mathrm{H}_{2} \mathrm{O}_{2}$ sensitivity of S. sclerotiorum decreased significantly $(P<0.05)$ compared with the nonamended PDA control.
\end{abstract}

At present, fungicides still play an essential and, in most cases, maybe the most important role in successful and effective plant disease management. When controlling fungal and oomycete plant pathogens by application of fungicides, a small amount of phytopathogens will inevitably be exposed to sublethal doses of fungicides. Exposure to sublethal doses of fungicides can occur under diverse circumstances, such as inappropriate fungicide applications, in combination with other fungicides with different active ingredient, and emergence of fungicide-resistant strains $(16,26)$. Normal doses of fungicides for sensitive phytopathogen strains are perhaps sublethal doses for fungicide-resistant strains. In most experiments, researchers are mainly interested in effects of normal (high) doses of fungicides on plant pathogens, and effects of sublethal doses of fungicides have been largely ignored.

In experiments to test in vitro sensitivity of Sclerotinia sclerotiorum (Lib.) de Bary to fungicide, we noticed that some isolates grew faster on potato dextrose agar (PDA) media amended with sublethal doses of fungicides than on fungicide-free PDA media. There have been some reports of plant pathogen growth and virulence stimulations by sublethal doses of fungicides or other chemicals (e.g., stimulation of Pythium damping-off of geranium by sublethal doses of mefenoxam; 18,19). The stimulations of sublethal doses of fungicides on phytopathogens probably belong to a phenomenon called hormesis. Hormesis is a toxicological concept characterized by low-dose stimulation and high-dose inhibition (6,17); hence, hormetic dose response curves are biphasic rather than monotonic (20). Extensive examinations of toxicological literature by Calabrese and Blain (11) indicated that hormetic dose responses are common across an enormously wide range of biological systems and stressors. The first scientific study

Corresponding author: F.-X. Zhu, E-mail: zhufuxing@mail.hzau.edu.cn

Accepted for publication 17 April 2014.

http://dx.doi.org/10.1094/PDIS-10-13-1059-RE

(c) 2014 The American Phytopathological Society of hormesis is deemed to be on growth stimulation of a wooddecaying fungus (Fomes officinalis) by extracts of western redcedar heartwood at low doses (17,32). Baraldi et al. (2) reported that low concentrations of thiabendazole amended in media could increase germination percentages of some but not all thiabendazole-resistant Penicillium expansum isolates. Landry et al. (23) observed significantly increased radial growth of Lyophyllum palustre (Peck) Singer on media amended with low concentrations of propamocarb compared with the fungicide-free control. With regard to oomycete plant pathogens, Phytophthora undulate could be stimulated by low doses of hymexazol (21). Metalaxyl-resistant $P$. infestans growth and virulence was stimulated by low concentrations of metalaxyl (37). Numerous reports of stimulation on plant pathogens indicated that resistant isolates or strains were probably more prone to be stimulated by sublethal doses of fungicides. Moorman (25) reported mycelial growth stimulations in some propamocarb and mefenoxam dualresistant strains of Pythium aphanidermatum, $P$. irregulare, and $P$. ultimum by propamocarb at $1 \mu \mathrm{g} / \mathrm{ml}$, and in some strains of $P$. aphanidermatum by propamocarb at $1,000 \mu \mathrm{g} / \mathrm{ml}$. More concerning about sublethal doses of fungicides is their virulence stimulatory effects on plant pathogens. Garzón et al. (18) reported that mycelial growth stimulatory effects of sublethal doses of mefenoxam on a mefenoxam-resistant isolate of $P$. aphanidermatum was about a $10 \%$ increase over the control whereas the percent stimulation on virulence of $P$. aphanidermatum on geranium seedlings was as high as $61 \%$. There is a possibility that virulence stimulations maybe relate to endogenous hydrogen peroxide $\left(\mathrm{H}_{2} \mathrm{O}_{2}\right)$ or oxalic acid in plant pathogens because these two compounds play a crucial role in fungal pathogenesis on host plants $(1,4,12,15,35)$.

The potential effects of fungicide hormesis are highly detrimental to agricultural productivity because they are beneficial to plant pathogens. Several fitness factors could be stimulated for the benefit of plant pathogens, including mycelial growth, spore germination, virulence, and toxin production $(16,17)$. When exposed to sublethal doses of the triazole fungicide prothioconazole, Fusarium graminearum produced more of the mycotoxin deoxyni- 
valenol than the control (1). Fungicide hormesis in plant pathogens has profound implications for integrated plant pathogen management and, thus, merits further study in detail.

S. sclerotiorum (Lib.) de Bary is a necrotrophic, omnivorous fungal plant pathogen with a broad host range of more than 400 plant species worldwide $(3,35)$, including economically important crops such as soybean, bean, sunflower, canola, and oilseed rape. Diseases caused by $S$. sclerotiorum have traditionally been difficult to control. Fungicides have been a major method for control of Sclerotinia diseases due to the lack of adequate levels of host resistance (4). The dicarboximide fungicide dimethachlon has been widely used to control Sclerotinia stem rot in China. Reduced sensitivity and high levels of resistance to dimethachlon in S. sclerotiorum isolates has been reported $(24,38)$. Up to the present, to our knowledge, there has been no report on stimulations of sublethal doses of a fungicide on mycelial growth in vitro or virulence in vivo of $S$. sclerotiorum.

The objectives of this study were to (i) determine in vitro stimulatory effects of sublethal doses of dimethachlon on mycelial growth of $S$. sclerotiorum, (ii) assess stimulatory effects of sublethal doses of dimethachlon on virulence of $S$. sclerotiorum in vivo, and (iii) explore possible mechanisms for stimulations on virulence by measuring sensitivity to $\mathrm{H}_{2} \mathrm{O}_{2}$ and production of oxalic acid of $S$. sclerotiorum exposed to sublethal doses of dimethachlon.

\section{Materials and Methods}

Isolates of S. sclerotiorum. Isolates of S. sclerotiorum were collected from oilseed rape plants with symptoms of Sclerotinia stem rot in China. Isolates LA50R, HF84R, and SCG7R were resistant to dimethachlon and the resistance was attained by consecutively growing dimethachlon-sensitive isolates LA50S, HF84S, and SCG7S on dimethachlon-amended PDA media for 25 generations. The other isolates were sensitive to dimethachlon.

Fungicides. Technical-grade dimethachlon (96.2\% active ingredient) was provided by Wenzhou Pesticide Co. Ltd. Dimethachlon was dissolved in $99.5 \%$ methanol to generate a stock solution $(1,000 \mu \mathrm{g} / \mathrm{ml})$ and the stock solution was stored in a refrigerator at $4^{\circ} \mathrm{C}$ for no longer than 2 weeks before being diluted for bioassay experiments.

Fungicide sensitivity determination. Fungicide sensitivity determination was performed according to Ma et al. (24), with slight modifications. Sclerotia of S. sclerotiorum were bisected, sterilized in $75 \%$ alcohol for $3 \mathrm{~min}$, transferred into $1 \%$ sodium hypochlorite solutions and sterilized for $3 \mathrm{~min}$, rinsed with sterile distilled water for $30 \mathrm{~s}$, and dried on sterilized filter paper. Halves of sclerotia were placed on PDA plates and incubated for 2 days at $26^{\circ} \mathrm{C}$ in a growth chamber (a 12-h photoperiod). Dimethachlon at a concentration of $5 \mu \mathrm{g} / \mathrm{ml}$ was used as the discriminatory dose to assess dimethachlon sensitivity or resistance in all isolates. Inverted mycelial plugs (6 $\mathrm{mm}$ in diameter) cut from actively growing margins of 2-day-old colonies were transferred to 9-cm petri dishes containing PDA media amended with dimethachlon at $5 \mu \mathrm{g} / \mathrm{ml}$, using fungicide-free PDA as the control. After $48 \mathrm{~h}$ of incubation at $26^{\circ} \mathrm{C}$ in a growth chamber, mycelial growth was measured in two perpendicular directions. Isolates that could grow with dimethachlon at $5 \mu \mathrm{g} / \mathrm{ml}$ were tentatively considered to be resistant to dimethachlon, whereas the completely inhibited ones were considered to be sensitive to dimethachlon. For eight isolates used in this study, the half maximal effective concentrations $\left(\mathrm{EC}_{50}\right)$ were determined as described below. PDA media amended with dimethachlon at $0,1,2,4,8,16,32,64$, and $128 \mu \mathrm{g} / \mathrm{ml}$ were used for the tentatively considered resistant isolates. PDA media amended with dimethachlon at $0,0.125,0.25,0.5,1.0$, and $2.0 \mu \mathrm{g} / \mathrm{ml}$ were used for sensitive isolates. Inverted mycelial plugs cut from fresh edges of 2-day-old colonies were transferred to 9-cm petri dishes containing dimethachlon-amended PDA media. After $48 \mathrm{~h}$ of incubation at $26^{\circ} \mathrm{C}$, two perpendicular diameters of each colony were measured (diameter of the plug was subtracted). $\mathrm{EC}_{50}$ values were calculated by linear regression of logarithms of fungicide concentrations versus probit values of radial growth inhibition percent- ages. Three replicates per concentration were used, and all tests were repeated twice.

Stimulatory effects of sublethal doses of dimethachlon on in vitro mycelial growth of $S$. sclerotiorum. Inverted mycelial plugs (6 $\mathrm{mm}$ in diameter) cut from fresh margins of 2-day-old colonies were transferred onto PDA media amended with various concentrations of dimethachlon. Dimethachlon concentrations were determined according to results of preliminary experiments to include at least one concentration that had a stimulatory effect on colony radial growth. Fungicide-free PDA and PDA amended with the same concentrations $(0.5$ and $1 \%)$ of methanol as in dimethachlonamended PDA were used as the controls. After $24 \mathrm{~h}$ of incubation at $26^{\circ} \mathrm{C}$ in a growth chamber, mycelial growth was measured in two perpendicular directions. Inhibitory or stimulatory effects relative to the control were calculated. (No stimulatory effects were observed for methanol treatment; therefore, fungicide-free PDA was used as the control in calculating stimulatory effects.) There were at least 8 replicates and all tests were repeated four times, thus with at least 24 replicates, in total, for each treatment.

Stimulatory effects of growing on dimethachlon-amended PDA on virulence of $S$. sclerotiorum on detached leaves of oilseed rape plants. Leaves of 12 to $15 \mathrm{~cm}$ in diameter were detached from 7- to 9-week-old oilseed rape plants growing in the greenhouse at $26^{\circ} \mathrm{C}$. The detached leaves were rinsed with sterile water, air dried on a clean bench, and transferred to $15-\mathrm{cm}$-diameter petri dishes lined with water-soaked, sterilized filter paper to maintain high humidity. Inverted mycelial plugs $(6 \mathrm{~mm}$ in diameter) cut from fresh margins of 2-day-old colonies growing on PDA media amended with dimethachlon at final concentrations of $0.5,2$, $8,16,20,22,30$, and $35 \mu \mathrm{g} / \mathrm{ml}$ were placed on the adaxial surface of detached leaves with the mycelium side down. Fungicide-free PDA amended with methanol were used as the control. The inoculated leaves were incubated in a growth chamber (photoperiod of $16 \mathrm{~h}$ of light and $8 \mathrm{~h}$ of darkness) at $26^{\circ} \mathrm{C}$, with relative humidity maintained over $85 \%$. Lesion diameters were measured perpendicularly after $48 \mathrm{~h}$ of incubation. The experiment was repeated three times with at least 24 replicates, in total, for each treatment.

Stimulatory effects of spraying sublethal doses of dimethachlon on virulence of $S$. sclerotiorum on detached leaves of oilseed rape plants. Oilseed rape leaves were selected and rinsed as described above. Dimethachlon solutions were prepared by diluting the dimethachlon stock solution with sterilized $0.1 \%$ Triton-100 water. Detached leaves were sprayed with dimethachlon solutions at concentrations of $0,1,5$, and $25 \mu \mathrm{g} / \mathrm{ml}$ by a hand-held sprayer. The treated leaves were air dried and inoculated on the adaxial surface with inverted mycelial plugs cut from fresh margins of 2-day-old colonies growing on fungicide-free PDA media. The inoculated leaves were incubated in a growth chamber (photoperiod of $16 \mathrm{~h}$ of light and $8 \mathrm{~h}$ of darkness) at $26^{\circ} \mathrm{C}$ with relative humidity maintained over $85 \%$. Lesion diameters were measured perpendicularly after $48 \mathrm{~h}$ of incubation. The experiment was repeated three times with at least 24 replicates, in total, for each treatment.

Stimulatory effects of spraying sublethal doses of dimethachlon on virulence of $S$. sclerotiorum on oilseed rape plants in pot experiments. Dimethachlon stock solutions were diluted to concentrations of 2,5 , and $25 \mu \mathrm{g} / \mathrm{ml}$ with sterilized water containing $0.1 \%$ Triton X-100. Approximately 8 -week-old oilseed rape plants growing in 20 -cm-diameter pots containing composite mixture of peat and soil (weight ratio, 1:1) were sprayed with dimethachlon at concentrations of 2,5 , and $25 \mu \mathrm{g} / \mathrm{ml}$. Oilseed rape plants sprayed with sterilized water containing $0.1 \%$ Triton X-100 and $0.5 \%$ methanol were used as the fungicide-free control. After about $1 \mathrm{~h}$ of air drying, leaves were inoculated on the adaxial surface by inverted mycelial plugs $(6 \mathrm{~mm}$ in diameter) cut from fresh margins of $48 \mathrm{~h}$-old colonies growing on fungicide-free PDA media. The inoculated plants were incubated in a growth chamber (photoperiod of $16 \mathrm{~h}$ of light and $8 \mathrm{~h}$ of darkness) at $26^{\circ} \mathrm{C}$ with relative humidity maintained over $85 \%$. Lesion diameters were measured perpendicularly after $48 \mathrm{~h}$ of incubation. The experiment 
was repeated three times with at least 12 replicates, in total, for each treatment.

Effects of dimethachlon on sensitivity of $S$. sclerotiorum to $\mathbf{H}_{2} \mathbf{O}_{2}$. Inverted mycelial plugs (6 $\mathrm{mm}$ in diameter) cut from fresh margins of 2-day-old colonies were transferred to PDA media amended with dimethachlon at $0 \mu \mathrm{g} / \mathrm{ml}$ (as the fungicide-free control) and $20 \mu \mathrm{g} / \mathrm{ml}$. After incubation in a growth chamber at $26^{\circ} \mathrm{C}$ for $48 \mathrm{~h}$, inverted mycelial plugs cut from fresh margins of these 48-h-old colonies were transferred to PDA media amended with $\mathrm{H}_{2} \mathrm{O}_{2}$ at 0,10 , and $100 \mu \mathrm{g} / \mathrm{ml}$. The radial growth was calculated by measuring two perpendicular diameters of each colony after incubating at $26^{\circ} \mathrm{C}$ for $24 \mathrm{~h}$. The experiment was repeated three times with at least 24 replicates, in total, for each treatment.

Effects of dimethachlon on oxalic acid production in S. sclerotiorum. Potato dextrose broth (PDB) media were amended with dimethachlon at $0,0.5,2,8,20$, and $30 \mu \mathrm{g} / \mathrm{ml}$. Four mycelial plugs (6 $\mathrm{mm}$ in diameter) of each isolate were inoculated into $200 \mathrm{ml}$ of PDB media amended with dimethachlon and then incubated at $26^{\circ} \mathrm{C}$ on an orbital shaker at $120 \mathrm{rpm}$. After $72 \mathrm{~h}$ of incubation, PDB media were centrifuged at $12,000 \mathrm{rpm}$ for $10 \mathrm{~min}$ and the supernatants were centrifuged at $12,000 \mathrm{rpm}$ for another $10 \mathrm{~min}$. The last supernatants were used to determine oxalic acid concentrations, according to the method described by Culbertson (14), with some modifications. Briefly, concentrations of oxalic acid were determined using a PROSTAR 230 high-performance liquid chromatograph (VARIAN Medical Systems Inc.) fitted with a Hypersil $C_{18}$ reversed-phase column ( 4.6 by $250 \mathrm{~mm}, 5 \mu \mathrm{m}$ ). Chromatographic conditions were as follows: column temperature, $26^{\circ} \mathrm{C}$; mobile phase, $0.01 \mathrm{~N} \mathrm{H}_{2} \mathrm{SO}_{4}$; flow rate of mobile phase, 1 $\mathrm{ml} / \mathrm{min}$; injection volume, $20 \mu \mathrm{l}$; and detection: $\mathrm{UV}, 210 \mathrm{~nm}$.

Data analysis. $\mathrm{EC}_{50}$ values were calculated by the procedure "Bioassay Analysis for Quantity Data" in DPS software (Data Processing System, ver. 7.05; Hangzhou RuiFeng Information Technology Co. Ltd.). Analysis of variance in SPSS (Statistical Product and Service Solutions, ver. 17.0; SPSS Inc.) and Fisher's least significant difference test $(P=0.05)$ were used to evaluate differences among different treatments. Each petri dish was defined as one experimental unit, with the arithmetic mean of two perpendicular measures of mycelial growth on PDA or lesion diameters on detached leaves as response. In pot experiments, the experimental unit was defined as each oilseed plant growing in one pot, with the arithmetic mean of lesion diameters as response. Graphs were created with Microsoft Excel (ver. 2010; Microsoft Corporation).

\section{Results}

Sensitivity to dimethachlon. The dimethachlon $\mathrm{EC}_{50}$ values of the three isolates HF84R, LA50R, and SCG7R were 9.31, 33.76,

Table 1. Dimethachlon sensitivity of eight isolates of Sclerotinia sclerotiorum

\begin{tabular}{lcc}
\hline Isolates $^{\mathbf{a}}$ & $\begin{array}{c}\text { Dimethachlon } \\
\text { sensitivity }^{\mathbf{b}}\end{array}$ & $\begin{array}{c}\text { Dimethachlon } \mathbf{E C}_{\mathbf{5 0}} \\
(\boldsymbol{\mu} \mathbf{g} / \mathbf{m l})\end{array}$ \\
\hline F78-1 & $\mathrm{S}$ & 0.02 \\
A53-3 & $\mathrm{S}$ & 0.06 \\
HF84S & $\mathrm{S}$ & 0.25 \\
LA50S & $\mathrm{S}$ & 0.19 \\
SCG7S & $\mathrm{S}$ & 0.71 \\
HF84R & $\mathrm{R}$ & 9.31 \\
LA50R & $\mathrm{R}$ & 33.76 \\
SCG7R & $\mathrm{R}$ & 83.54 \\
\hline
\end{tabular}

a In total, 58 isolates, including 3 dimethachlon-resistant isolates and 55 sensitive isolates, were used in this study. Only eight isolates were listed here.

${ }^{\mathrm{b}} \mathrm{S}$ represents dimethachlon-sensitive isolates, whose mycelia growth was completely inhibited on potato dextrose agar (PDA) media amended with dimethachlon at $5 \mu \mathrm{g} / \mathrm{ml}$, and the sensitivity was corroborated by half maximal effective concentration $\left(\mathrm{EC}_{50}\right)$ values lower than $1 \mu \mathrm{g} / \mathrm{ml}$. $\mathrm{R}$ represents dimethachlon-resistant isolates, whose mycelia could grow on PDA media amended with dimethachlon at $5 \mu \mathrm{g} / \mathrm{ml}$, and the resistance was corroborated by $\mathrm{EC}_{50}$ values higher than $5 \mu \mathrm{g} / \mathrm{ml}$. and $83.54 \mu \mathrm{g} / \mathrm{ml}$, respectively (Table 1 ). These results corroborated that isolates LA50R, HF84R, and SCG7R were resistant to dimethachlon. The other 55 isolates were sensitive to dimethachlon and their $\mathrm{EC}_{50}$ values for dimethachlon were lower than $1.0 \mu \mathrm{g} / \mathrm{ml}$.

Stimulatory effects of sublethal doses of dimethachlon on in vitro mycelial growth of $S$. sclerotiorum. All three dimethachlonresistant isolates grew faster on PDA amended with sublethal concentrations of dimethachlon than on PDA amended only with methanol as the control. The largest growth increases were 12.24 to $17.24 \%$ compared with the control. Dimethachlon at concentrations from 0.5 to $4 \mu \mathrm{g} / \mathrm{ml}$ showed stimulatory effects on mycelial growth of dimethachlon-resistant isolate HF84R and LA50R (Fig. 1). The largest growth increase for isolate HF84R was obtained with dimethachlon at $1 \mu \mathrm{g} / \mathrm{ml}$ and the largest growth increase for isolate LA50R was obtained with dimethachlon at $2 \mu \mathrm{g} / \mathrm{ml}$.

Stimulatory effects of growing on dimethachlon-amended PDA on virulence of $S$. sclerotiorum on detached leaves of oilseed rape plants. After growing on dimethachlon-amended PDA media, increases in virulence on detached leaves of oilseed

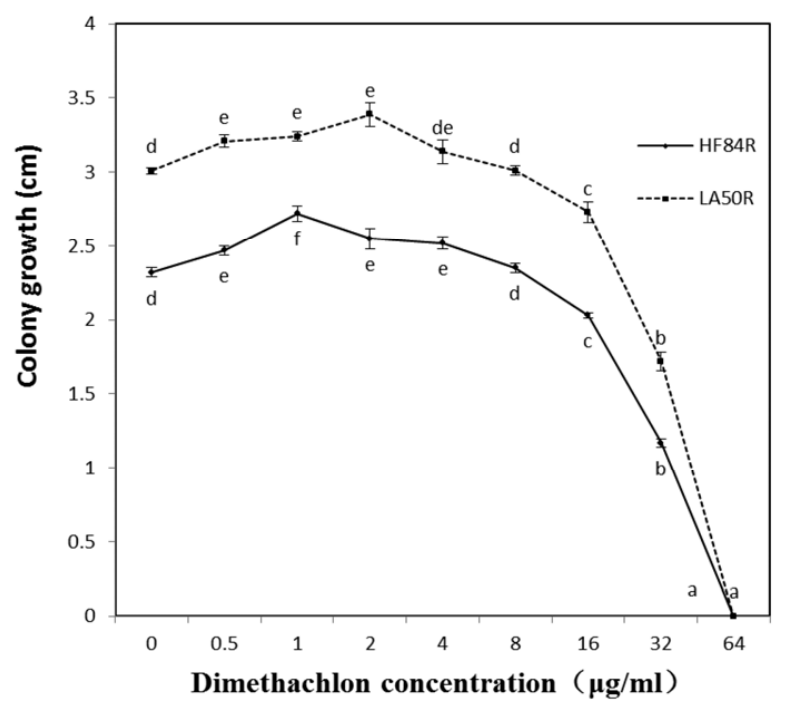

Fig. 1. Mycelial growth of dimethachlon-resistant isolates HF84R and LA50R on dimethachlon-amended potato dextrose agar media. Each data point in the figure represented the mean value and standard error of 24 replicates. Different letters on the same line represented statistically significant differences $(P=0.05)$ in arithmetic means of colony growth among different treatments.

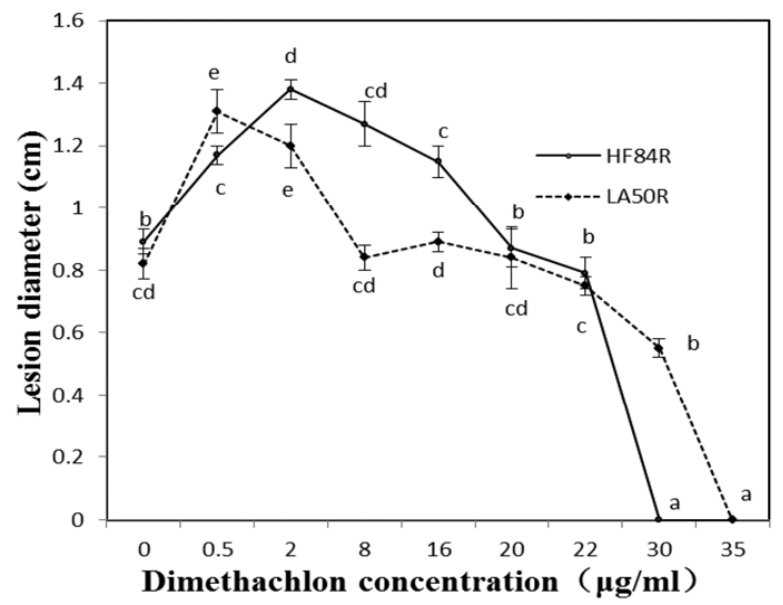

Fig. 2. Virulence of isolates HF84R and LA50R after growing on dimethachlonamended potato dextrose agar for $48 \mathrm{~h}$ on detached leaves of oilseed rape plants. Each data point in the figure represented the mean value and standard error of 24 replicates. Different letters on the same line represented statistically significant differences $(P=0.05)$ in arithmetic means of lesion diameters among different treatments. 
rape plants were statistically significant for dimethachlon-resistant isolates. All three dimethachlon-resistant isolates showed virulence stimulation responses after growing on PDA amended with dimethachlon at 0.5 to $2 \mu \mathrm{g} / \mathrm{ml}$. The maximum stimulations on virulence ranged from 42.40 to $59.80 \%$, which were much higher than stimulations on mycelial growth on PDA media. For isolate HF84R, virulence stimulation was found at dimethachlon concentrations from 0.5 to $16 \mu \mathrm{g} / \mathrm{ml}$ and the maximum stimulation was obtained with dimethachlon at $2 \mu \mathrm{g} / \mathrm{ml}$ (Fig. 2).

Stimulatory effects of spraying sublethal doses of dimethachlon on virulence of $S$. sclerotiorum on detached leaves of oilseed rape plants. Of the 55 sensitive isolates tested, only 13 (or $23.64 \%$ ) of the sensitive isolates displayed virulence stimulatory responses to sublethal doses of dimethachlon sprayed on detached leaves of oilseed rape plants under the present experimental conditions (Table 2). For dimethachlon-resistant isolates LA50R and HF48R, the maximum stimulation effects on virulence were 57.14 and $17.20 \%$, respectively, obtained at a dimethachlon concentration of $25 \mu \mathrm{g} / \mathrm{ml}$. For sensitive isolates such as HF84S and LA50S, dimethachlon at $1 \mu \mathrm{g} / \mathrm{ml}$ showed stimulatory effects on virulence whereas dimethachlon at $25 \mu \mathrm{g} / \mathrm{ml}$ showed inhibitory rather than stimulatory effects on virulence compared with the control (Fig. 3).

Stimulatory effects of spraying sublethal doses of dimethachlon on virulence of $S$. sclerotiorum on oilseed rape plants in pot experiments. For both sensitive isolates and dimethachlonresistant isolates, significant $(P<0.05)$ stimulations on virulence were obtained with dimethachlon at $2 \mu \mathrm{g} / \mathrm{ml}$ (Fig. 4). Virulence stimulation magnitude was 19.28 to $62.99 \%$. Dimethachlon at 5 $\mu \mathrm{g} / \mathrm{ml}$ did not show significant differences in lesion diameters compared with the control. Dimethachlon at $25 \mu \mathrm{g} / \mathrm{ml}$ completely inhibited virulence of sensitive isolate LA50S, and virulence of resistant isolate LA50R was inhibited by only $40.71 \%$ at the same concentration.

Effects of dimethachlon on sensitivity of $S$. sclerotiorum to $\mathbf{H}_{2} \mathbf{O}_{2}$. After growing on dimethachlon-amended PDA media, sensitivity of $S$. sclerotiorum to $\mathrm{H}_{2} \mathrm{O}_{2}$ decreased significantly $(P<$ $0.05)$ compared with the fungicide-free control. Growth of isolate LA50R without dimethachlon-pretreatment was completely inhibited on $\mathrm{H}_{2} \mathrm{O}_{2}$-amended PDA at $100-\mu \mathrm{g} / \mathrm{ml}$. However, after growing on PDA amended with dimethachlon at $20-\mu \mathrm{g} / \mathrm{ml}$ for 2 days, LA50R could grow on $100-\mu \mathrm{g} / \mathrm{ml}-\mathrm{H}_{2} \mathrm{O}_{2}$-amended PDA, although the growth was inhibited by $64.05 \%$ compared with growth on $\mathrm{H}_{2} \mathrm{O}_{2}$-free PDA (Fig. 5). Mycelial growth of LA50R pretreated with dimethachlon at $20-\mu \mathrm{g} / \mathrm{ml}$ on $10-\mu \mathrm{g} / \mathrm{ml}^{-} \mathrm{H}_{2} \mathrm{O}_{2}$-amended PDA increased significantly $(P<0.05)$ compared with that without dimethachlon pretreatment. These results indicated that growing on dimethachlon-amended PDA could enhance tolerance of $S$. sclerotiorum to $\mathrm{H}_{2} \mathrm{O}_{2}$ stress.

Effects of dimethachlon on oxalic acid production in $\mathrm{S}$. sclerotiorum. Dimethachlon at final concentrations of 0.5 to $30 \mu \mathrm{g} / \mathrm{ml}$ did not stimulate oxalic acid production in isolate HF84R growing in PDB media (Fig. 6). On the contrary, oxalic acid production decreased significantly at these concentrations of dimethachlon. This result was similar to that of isolate LA50R (data not shown).

\section{Discussion}

This study demonstrated that sublethal doses of dimethachlon could stimulate mycelial growth of $S$. sclerotiorum on PDA media and significantly increase virulence on oilseed rape plants.
Hormesis may be involved in these stimulations. Hormesis is a biphasic dose-response phenomenon characterized by stimulations at low doses and inhibitions at high doses of a stressor. In order to detect hormesis scientifically, some experimental design requirements, such as no observed adverse effect level (NOAEL), should be determined and evenly distributed doses below the NOAEL should be tested to provide enough data for hormesis (16). The NOAEL value can be estimated by Brain-Cousens nonlinear regression model (5), as described by Schabenberger et al. (30). Analysis of the hormesis database, which contained nearly 9,000 dose responses by Calabrese and Blain (11), indicated that hormetic effects tended to be modest, commonly differing from the control by about 30 to $60 \%$, and the hormetic zone occurred immediately below the NOAEL and its width often extended 10- to 20fold. The present study demonstrated that the largest stimulatory effects on virulence on detached leaves of oilseed rape plants by sublethal doses of dimethachlon ranged from 17.20 to $59.80 \%$ across different isolates, and the range of dimethachlon concentrations having stimulating effects was approximately 20-fold for resistant isolates (Table 2). All these data indicated that the stimulatory effects of sublethal doses of dimethachlon on virulence of $S$. sclerotiorum might be hormesis. More evenly distributed concentrations of dimethachlon below the NOAEL should be tested to verify whether this was hormesis.

There have been many debates in the scientific community about the generality of hormesis. In 2005, Calabrese and Blain published the assessment results of the hormesis database that contained over 5,000 dose responses (10). The findings indicated that hormetic dose responses were broadly generalizable, as observed in a broad range of biological models (from microorganisms to plants to mammals), over numerous and diverse measured endpoints, across a wide range of chemical classes, and independent of mechanism. They concluded that hormetic dose responses were reproducible and common across diverse biological systems. However, after extensive literature review, Mushak (27) stated that the literature

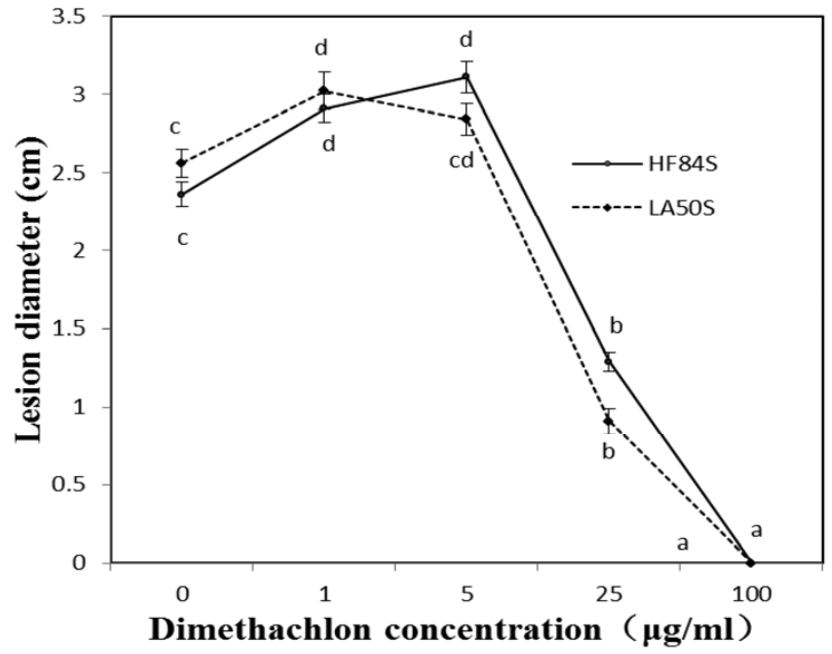

Fig. 3. Stimulatory virulence effects of spraying sublethal doses of dimethachlon on detached leaves of oilseed rape plants. Each data point in the figure represented the mean value and standard error of 24 replicates. Different letters on the same line represented statistically significant differences $(P=0.05)$ in arithmetic means of lesion diameters among different treatments.

Table 2. Percentage of isolates exhibiting stimulatory virulence responses to sublethal doses of dimethachlon sprayed on detached leaves of oilseed rape plants

\begin{tabular}{lcccc}
\hline $\begin{array}{l}\text { Sensitivity to } \\
\text { dimethachlon }\end{array}$ & $\begin{array}{c}\text { Total number } \\
\text { of isolates }\end{array}$ & $\begin{array}{c}\text { Number of isolates exhibiting } \\
\text { stimulatory responses }^{\mathbf{a}}\end{array}$ & $\begin{array}{c}\text { Isolates exhibiting stimulatory } \\
\text { responses }(\%)\end{array}$ & $\begin{array}{c}\text { Dimethachlon concentrations with } \\
\text { stimulating effects }(\boldsymbol{\mu g} / \mathbf{m l})\end{array}$ \\
\hline Sensitive & 55 & 13 & 23.64 & $1.0 \approx 5.0$ \\
Resistant & 3 & 3 & 100.00 & $1.0 \approx 25.0$ \\
\hline
\end{tabular}

${ }^{a}$ Stimulatory responses were recognized when there was at least one dose of dimethachlon causing statistically significant increases (stimulations) $(P=$ 0.05 ) in virulence (disease lesions) on detached leaves of oilseed rape plants. There were at least 24 replicates, in total, for each treatment. 
selected for generalizability analyses had not been systematically preevaluated as a scientifically reliable representation of the hormesis frequency in nature. Simultaneous estimates of the frequency of nonhormetic dose-response relationships required for reliable determination of hormesis frequency were not done in these analyses. No evidence in the available literature was found to show that hormesis was generalizable in the natural or experimental worlds (27). Chemical hormesis has been mainly evaluated for isolated endpoints (22). Hormesis determination relies heavily on statistical analysis of the measured endpoint. Zeiger et al. (36) did not believe that it was enough to look at the calculated $P$ value for the chosen statistic parameter when attempting to determine whether a biological effect such as hormesis was present. Nonetheless, the remarkable consistency of the quantitative features of hormetic dose responses across an enormously wide range of biological systems and endpoints indicates that hormesis is broadly generalizable.

Detection of hormesis is a challenging work. Evaluation of data is very important for testing hormesis. Crump (13) proposed the criteria for evaluating hormesis as follows: strength of evidence, soundness of data, consistency, and biological plausibility. The curves of S. sclerotiorum mycelial growth on PDA and virulence on detached leaves of oilseed rape plants in response to various concentrations of dimethachlon showed similarity to typical hormetic curves and had the same biphasic characteristic. Our results showed that all three dimethachlon-resistant isolates and only $23.64 \%$ of sensitive isolates displayed stimulatory responses to sublethal doses of dimethachlon. It seemed that fungicide-resistant isolates were more likely to exhibit hormetic responses. It should be noted that the failure to detect stimulatory responses in some sensitive isolates might have been due to inadequate sublethal concentrations of dimethachlon in the current experiment. If more sublethal concentrations were tested, stimulatory growth might be detected in more isolates. It was less likely to discover hormetic responses in sensitive isolates than in resistant isolates because there would have been fewer concentrations below the NOAEL. There were examples indicating that some species or individuals might lack the capacity to produce the low-dose stimulatory response (9). The differential responses to low doses of chemicals in different organisms need further investigations.

The obvious stimulations on mycelial growth in vitro and significantly increased virulence on oilseed rape plants have much relevance to plant disease management. Even though only a small percentage of isolates can exhibit stimulatory responses to sublethal doses of fungicides, there is a high possibility of increased plant disease incidence and severity in the field because contacting sublethal doses of fungicides is inevitable due to inappropriate fungicide applications and low-dose applications to reduce costs, among other reasons (16). In fact, it has been known that, in some



circumstances, Sclerotinia stem rot disease caused by S. sclerotiorum in oilseed rape plants will become aggravated after application of fungicides (e.g., carbendazim; personal communication). This may be due to virulence stimulatory effects of sublethal doses of fungicides on some isolates, especially on fungicide-resistant isolates. At present, the effects of sublethal doses of fungicides on plant pathogens are awaiting their due attention from the scientific community.

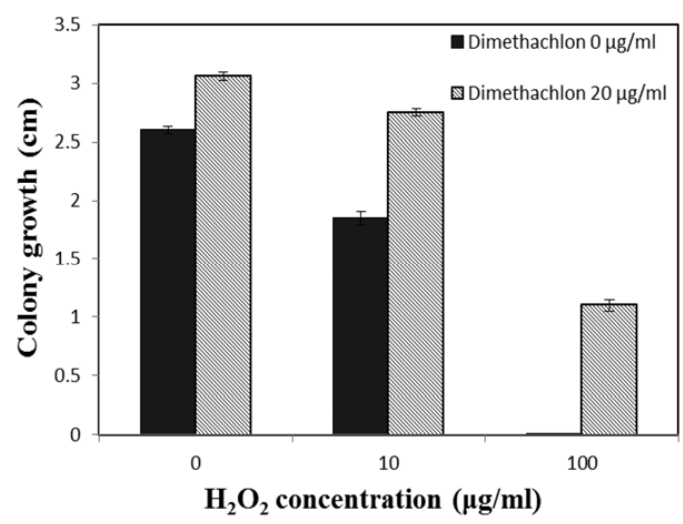

Fig. 5. Effects of pretreatment with dimethachlon on sensitivity of isolate LA50R to $\mathrm{H}_{2} \mathrm{O}_{2}$. In this figure, "Dimethachlon $0 \mu \mathrm{g} / \mathrm{ml}$ " denoted that isolate LA50R was grown on fungicide-free potato dextrose agar (PDA) as the control. "Dimethachlon 20 $\mu \mathrm{g} / \mathrm{ml}$ " denoted that isolate LA50R was pretreated with dimethachlon (i.e., grown for 2 days on PDA media amended with dimethachlon at $20 \mu \mathrm{g} / \mathrm{ml}$ ). Each column in the figure represented the mean value and standard error of 24 replicates.

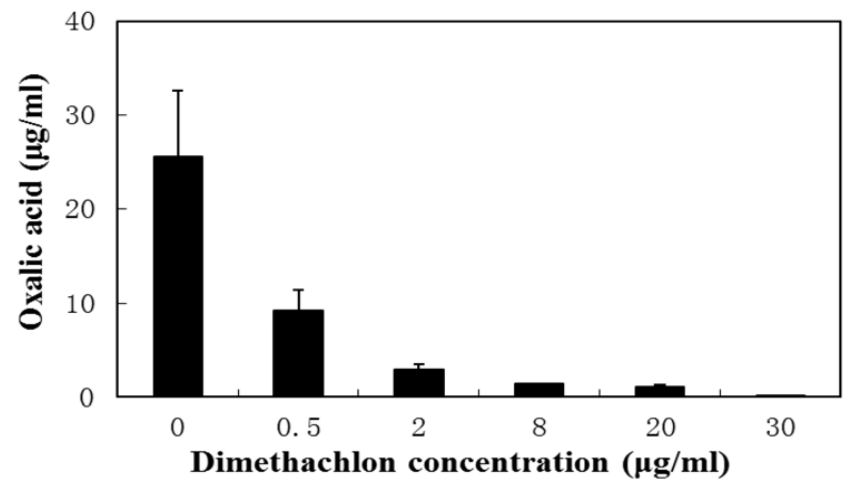

Fig. 6. Effects of dimethachlon on oxalic acid production in isolate HF84R in potato dextrose broth. Each column in the figure represented the mean value and standard error of six replicates.

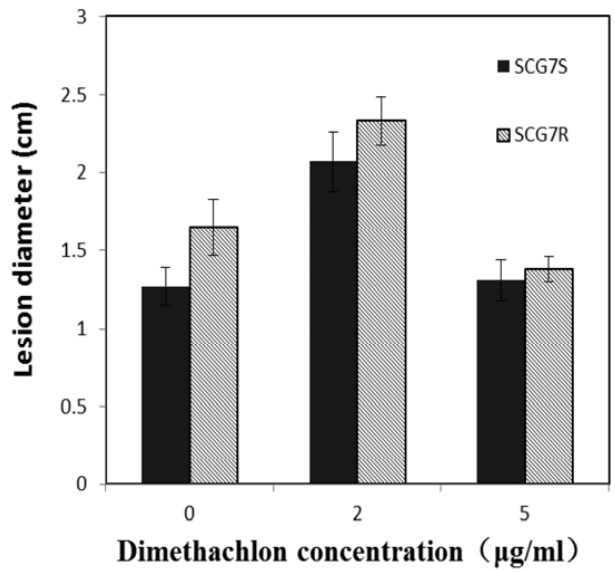

Fig. 4. Stimulatory virulence effects of spraying sublethal doses of dimethachlon in pot experiments. Isolates LA50R and SCG7R were derived from isolates LA50S and SCG7S, respectively, by consecutive selections on dimethachlon-amended potato dextrose agar for 25 generations. Each column in the figure represented the mean value and standard error of 12 replicates. 
Stimulations on mycelial growth and virulence by sublethal doses of fungicides have been observed in ascomycetes (1) and basidiomycetes $(16,23)$ as well as in oomycetes $(18)$. The potential effects of fungicide hormesis are highly detrimental, because they may result in greater disease incidence and severity, larger crop losses, and higher mycotoxin levels in grains (1). More studies are needed to assess the prevalence and quantitative features of hormesis in plant pathogens. Understanding the qualitative and quantitative characteristics of fungicide hormesis and subsequent integration of the hormesis concept into plant disease management will undoubtedly improve fungicide application efficiency and enhance crop productivity.

Recently, Calabrese (7) gave a comprehensive review on the mechanisms of hormesis, particularly for those mediated via receptor or cell signaling pathways. Protecting the organism from injury via adaptive and preconditioning stress responses or overcompensation for a disruption of homeostasis has been claimed to be the mechanistic basis for chemical hormesis (8). However, some scientists argued that overcompensation for homeostatic preservation in biological systems was not a necessary quantitative condition for such preservation. Sufficient rather than excessive compensation would be more biologically and physiologically logical, and compensation sufficiency could occur within the threshold doseresponse model (28). The diversity of models for hormetic responses suggests that the mechanisms may not be the same for different systems (17). In this study, virulence of isolate HF84R on detached leaves with dimethachlon at $8 \mu \mathrm{g} / \mathrm{ml}$ was stimulated by $14.73 \%$ after $48 \mathrm{~h}$ of inoculation and by $24.57 \%$ after $72 \mathrm{~h}$ of inoculation. For isolate LA50R under the same conditions, the virulence stimulation effect decreased from $36.04 \%$ after $48 \mathrm{~h}$ of inoculation to $21.24 \%$ after $72 \mathrm{~h}$ of inoculation. These experimental results suggested that the hormetic responses were differential for different isolates of even the same species of plant pathogen. The underlying mechanisms for hormetic responses are complex and remain to be elucidated in the future.

Previous studies demonstrated that the $S$. sclerotiorum growth rate was markedly reduced by $\mathrm{H}_{2} \mathrm{O}_{2}$ at concentrations between 12.5 and $20 \mathrm{mM}$ and was completely inhibited by $\mathrm{H}_{2} \mathrm{O}_{2}$ at concentrations higher than $20 \mathrm{mM}$ (34). Sublethal doses of the triazole fungicide prothioconazole could induce production of $\mathrm{H}_{2} \mathrm{O}_{2}$ in $\mathrm{F}$. graminearum (1). $\mathrm{H}_{2} \mathrm{O}_{2}$ inducement by sublethal doses of a fungicide may be one of the reasons for reduced sensitivity to $\mathrm{H}_{2} \mathrm{O}_{2}$ after $S$. sclerotiorum growth on fungicide-amended PDA. Whether sublethal doses of dimethachlon can induce $\mathrm{H}_{2} \mathrm{O}_{2}$ production or not in $S$. sclerotiorum remains to be investigated in the future. One of the earliest and most common resistance responses mounted by plant tissue against an invading microbe is the oxidative burst, a controlled release of $\mathrm{O}_{2}{ }^{-}$and $\mathrm{H}_{2} \mathrm{O}_{2}$ at the point of pathogen challenge (35). Infection of bean and tomato leaves by Botrytis cinerea resulted in massive accumulation of $\mathrm{H}_{2} \mathrm{O}_{2}$, both in the plant plasma membrane and in the extracellular sheath covering the surface of fungal hyphae $(31,33)$. The reduced sensitivity of $S$. sclerotiorum to $\mathrm{H}_{2} \mathrm{O}_{2}$, at least in theory, could increase its virulence to host plants. Oxalic acid production has been associated with virulence of some Sclerotinia spp. $(15,29)$. Oxalic acid could suppress the oxidative burst of the host plant (12), enhance activities of cellwall-degrading enzymes by reducing extracellular $\mathrm{pH}$, and had other functions conducive to virulence (4). This study clearly demonstrated that stimulatory effects of sublethal doses of dimethachlon on virulence were not due to an increase in oxalic acid secretion. Factors other than oxalic acid should be involved in virulence stimulations of $S$. sclerotiorum by sublethal doses of dimethachlon.

\section{Acknowledgments}

This study was sponsored by National Natural Science Foundation of China (number 31371964) and the Special Fund for Agro-scientific Research in the Public Interest (number 201103016). We thank D. Jiang of Huazhong Agricultural University for kindly providing S. sclerotiorum isolates and $\mathrm{X}$. Sun of Wuhan University for suggestions on this manuscript.

\section{Literature Cited}

1. Audenaert, K., Callewaert, E., Höfte, M., Saeger, S. D., and Haesaert, G. 2010. Hydrogen peroxide induced by the fungicide prothioconazole triggers deoxynivalenol (DON) production by Fusarium graminearum. BMC Microbiol. 10:112. doi:10.1186/1471-2180-10-112

2. Baraldi, E., Mari, M., Chierici, E., Pondrelli, M., Bertolini, P., and Pratella, G. C. 2003. Studies on thiabendazole resistance of Penicillium expansum of pears: Pathogenic fitness and genetic characterization. Plant Pathol. 52:362370.

3. Boland, G. J., and Hall, R. 1994. Index of plant hosts of Sclerotinia sclerotiorum. Can. J. Plant Pathol. 16:93-108.

4. Bolton, M. C., Thomma, B. P. H. J., and Nelson, B. D. 2006. Sclerotinia sclerotiorum (Lib.) de Bary: Biology and molecular traits of a cosmopolitan pathogen. Mol. Plant Pathol. 7:1-16.

5. Brain, P., and Cousens, R. 1989. An equation to describe dose responses where there is stimulation of growth at low doses. Weed Res. 29:93-96.

6. Calabrese, E. J. 2008. Hormesis: Why it is important to toxicology and toxicologists. Environ. Toxicol. Chem. 27:1451-1474.

7. Calabrese, E. J. 2013. Hormetic mechanisms. Crit. Rev. Toxicol. 43:580606.

8. Calabrese, E. J., Bachmann, K. A., Bailer, A. J., Bolger, P. M., Borak, J., Cai, L., Cedergreen, N., Cherian, M. G., Chiueh, C. C., Clarkson, T. W., Cook, R. R., Diamond, D. M., Doolittle, D. J., Dorato, M. A., Duke, S. O. Feinendegen, L., Gardner, D. E., Hart, R. W., Hastings, K. L., Hayes, A. W., Hoffmann, G. R., Ives, J. A., Jaworowski, Z., Johnson, T. E., Jonas, W. B., Kaminski, N. E., Keller, J. G., Klaunig, J. E., Knudsen, T. B., Kozumbo, W. J., Lettieri, T., Liu, S. Z., Maisseu, A., Maynard, K. I., Masoro, E. J., McClellan, R. O., Mehendale, H. M., Mothersill, C., Newlin, D. B., Nigg, H. N., Oehme, F. W., Phalen, R. F., Philbert, M. A., Rattan, S. I., Riviere, J. E., Rodricks, J., Sapolsky, R. M., Scott, B. R., Seymour, C., Sinclair, D. A., Smith-Sonneborn, J., Snow, E. T., Spear, L., Stevenson, D. E., Thomas, Y., Tubiana, M., Williams, G. M., and Mattson, M. P. 2007. Biological stress response terminology: Integrating the concepts of adaptive response and preconditioning stress within a hormetic dose-response framework. Toxicol. Appl. Pharmacol. 222:122-128.

9. Calabrese, E. J., and Baldwin, L. A. 2002. Hormesis and high-risk groups. Regul. Toxicol. Pharmacol. 35:414-428.

10. Calabrese, E. J., and Blain, R. B. 2005. The occurrence of hormetic dose responses in the toxicological literature, the hormesis database: An overview. Toxicol. Appl. Pharmacol. 202:289-301.

11. Calabrese, E. J., and Blain, R. B. 2011. The hormesis database: The occurrence of hermetic dose-responses in the toxicological literature. Regul. Toxicol. Pharmacol. 61:73-81.

12. Cessna, S. G., Sears, V. E., Dickman, M. B., and Low, P. S. 2000. Oxalic acid, a pathogenicity factor for Sclerotinia sclerotiorum, suppresses the oxidative burst of the host plant. Plant Cell 12:2191-2199.

13. Crump, K. 2001. Evaluating the evidence for hormesis: A statistical perspective. Crit. Rev. Toxicol. 31:669-679.

14. Culbertson, B. J., Furumo, N. C., and Daniel, S. L. 2007. Impact of nutritional supplements and monosaccharides on growth, oxalate accumulation, and culture $\mathrm{pH}$ by Sclerotinia sclerotiorum. FEMS Microbiol. Lett. 270:132-138

15. Dutton, M. V., and Evans, C. S. 1996. Oxalate production by fungi: Its role in pathogenicity and ecology in the soil environment. Can. J. Microbiol. 42:881-895.

16. Flores, F. J., and Garzón, C. D. 2013. Detection and assessment of chemical hormesis on the radial growth in vitro of oomycetes and fungal plant pathogens. Dose-Response 11:361-373.

17. Garzón, C. D., and Flores, F. J. 2013. Hormesis: Biphasic dose-responses to fungicides in plant pathogens and their potential threat to agriculture. In: Fungicides-showcases of integrated plant disease management from around the world. p311-328. INTECH. http://cdn.intechopen.com/pdfs-wm/44734.pdf

18. Garzón, C. D., Molineros, J. E., Yanez, J. M., Flores, F. J., Jimenez-Gasco, M. M., and Moorman, G. W. 2011. Sublethal doses of mefenoxam enhance Pythium damping-off of geranium. Plant Dis. 95:1233-1238.

19. Garzón, C. D., Moorman, G. W., Yanez, J. M., Molineros, J. E., Leonard, R. C., and Jimenez-Gasco, M. M. 2008. Low-doses of fungicides have a stimulatory effect on Pythium spp. in vitro and in planta. (Abstr.) Phytopathology 98:S58.

20. Hoffmann, G. R. 2009. A perspective on the scientific, philosophical, and policy dimensions of hormesis. Dose-Response 7:1-51.

21. Kato, S., Coe, R., New, L., and Dick, M. 1990. Sensitivities of various oomycetes to hymexazol and metalaxyl. J. Gen. Microbiol. 136:2127-2134.

22. Kendig, E. L., Le, H. H., and Belcher, S. M. 2010. Defining hormesis: Evaluation of a complex concentration response phenomenon. Int. J. Toxicol. 29:235-246.

23. Landry, J., Martinez, C., and Rochefort, L. 2011. The use of fungicide Nova to mitigate infection of Sphagnum by parasitic fungi in the greenhouse. Botany 89:655-661

24. Ma, H. X., Feng, X. J., Chen, Y., Chen, C. J., and Zhou, M. G. 2009. Occurrence and characterization of dimethachlon insensitivity in Sclerotinia scle rotiorum in Jiangsu Province of China. Plant Dis. 93:36-42.

25. Moorman, G. W., and Kim, S. H. 2004. Species of Pythium from green- 
houses in Pennsylvania exhibit resistance to propamocarb and mefenoxam. Plant Dis. 88:630-632.

26. Moorman, G. W., and Lease, R. J. 1992. Benzimidazole- and dicarboximide-resistant Botrytis cinerea from Pennsylvania greenhouses. Plant Dis. 76:477-480.

27. Mushak, P. 2013. How prevalent is chemical hormesis in the natural and experimental worlds? Sci. Total Environ. 443:573-581.

28. Mushak, P. 2013. Limits to chemical hormesis as a dose-response model in health risk assessment. Sci. Total Environ. 443:643-649.

29. Pierson, P. E., and Rhodes, L. H. 1992. Effect of culture medium on the production of oxalic acid by Sclerotinia trifoliorum. Mycologia 84:467-469.

30. Schabenberger, O., Tharp, B. E., Kells, J. J., and Penner, D. 1999. Statistical tests for hormesis and effective dosages in herbicide dose-response. Agron. J. 91:713-721.

31. Schouten, A., Tenberge, K. B., Vermeer, J., Stewart, J., Wagemakers, L., and Williamson, B. 2002. Functional analysis of an extracellular catalase of $\mathrm{Bo}$ trytis cinerea. Mol. Plant Pathol. 3:227-238.

32. Southam, C. M., and Erhlich, J. 1943. Effects of extracts of western redcedar heartwood on certain wood-decaying fungi in culture. Phytopathology
33:517-524.

33. van Kan, J. A. L. 2006. Licensed to kill: The lifestyle of a necrotrophic plant pathogen. Trends Plant Sci. 11:247-253.

34. Walz, A., Zingen-Sell, I., Theisen, S., and Kortekamp, A. 2008. Reactive oxygen intermediates and oxalic acid in the pathogenesis of the necrotrophic fungus Sclerotinia sclerotiorum. Eur. J. Plant Pathol. 120:317-330.

35. Williams, B., Kabbage, M., Kim, H. J., Britt, R., and Dickman, M. B. 2011. Tipping the balance: Sclerotinia sclerotiorum secreted oxalic acid suppresses host defenses by manipulating the host redox environment. PLoS Pathog. 7:e1002107. doi:10.1371/journal.ppat.1002107

36. Zeiger, E., and Hoffmann, G. R. 2012. An illusion of hormesis in the Ames test: Statistical evidence is not equivalent to biological significance. Mutat. Res. 746:89-93.

37. Zhang, S., Panaccione, S. G., and Gallegly, M. E. 1997. Metalaxyl stimulation of growth of isolates of Phytophthora infestans. Mycologia 89:289292.

38. Zhou, F., Zhang, X. L., Li, J. L., and Zhu, F. X. 2014. Dimethachlon resistance in Sclerotinia sclerotiorum in China. Plant Dis. In press. http://dx.doi.org/10.1094/PDIS-10-13-1072-RE 